cake differences of two or even three per cent. are not unusual in the percentage of oil found by different analysts.

I have submitted this method to a long series of tests in my laboratory during the past winter and I have been so satisfied with the accuracy and uniformity of the results obtained by it and with the simplicity and rapidity of the whole process that I cordially recommend it to agricultural chemists in America and especially to those who have small iaboratories and a large number of samples to analyze.

The apparatus is not costly and I hope the description I have given of it is sufficiently clear to enable it to be easily understood and imitated.

\title{
ON THE DETERMINATION OF NITRATES IN POTABLE WATER.
}

BV AUGLSTUS H, GILL.

keceived lankary 16, 1804 .

PART T.

THE remark of Tiemann, that the estimation of no other substance has so constantly occupied the attention of the analytical chemists of the last decade, to the extent to which the determination of nitric acid has engaged (literally "enchained") them, will, I think, be appreciated by all those who have worked upon the subject. Perhaps no determination requires more care, or occasions more trouble in its execution, or is more unsatisfactory when finished, than the one in question.

There are two general modes of procedure:

a. The direct estimation, and

b. The reduction to ammonia.

a. DIRECT ESTIMATION.

The direct processes which have been found to give the best results are those of Schulz-Tiemann1, ${ }^{2}$ Schloesing-Reichardt, ${ }^{3}$ Crum-Lunge, ${ }^{4}$ and Marx-Trommsdorf. ${ }^{5}$ These are only appli-

1 Tiemann Gaertner "Wasseranalyse" 3rd. Ed'n., p. I6s.

2 Ber, d. chem Ges., 6, 104 I.

8 Ztschr. anal. Chem., 9, 24.

4 Phil. Mag., (3), 30, 426 .

s Ztschr. anal. Chem., 9.17!. 
cable where relatively large amounts of nitrate are to be estimated, over 0.6 parts of nitrogen as nitrate per $100, \infty 00$, and even with this quantity two or three hundred $c c$. of the water must be evaporated for use. This concentration is objectionable as it offers an opportunity for the oxidation of nitrites and their decomposition with loss of nitrogen, ${ }^{1}$ and also for the oxidation of organic matter, and where many determinations are to be made it is very troublesome.

There is too, a decided advantage in estimating the nitrogen contents immediately upon receipt of the sample, and if possible, upon the water as received. This has been pointed out by $\mathrm{Tidy}^{2}$ in reference to the carbon contents of a water, and applies with still greater force to the nitrogen which is more susceptible to change.

Another direct method is the phenolsulphonic acid process; it originated with Sprengel ${ }^{3}$ and apparently fell into disuse until it was again brought to notice by Grandval and Lajoux." Since then articles upon it have appeared by Fox, ${ }^{5}$ Johnson, ${ }^{6}$ Lindo, ${ }^{7}$ Smith, ${ }^{8}$ Bartram, ${ }^{9}$ and Hazen and Clark. ${ }^{10}$

The two latter have criticised the process severely, and it seems to me unfairly, and it was with the idea of seeing how far

$1 \mathrm{NH}_{4} \mathrm{NO}_{2}=2 \mathrm{H}_{2} \mathrm{O}+\mathrm{N}_{2}$.

$2 J$. Chem. Soc., 35, 53 and 97.

8 Pogg. Ann., $121,188$.

4 Compt. rend., $101,62$.

b Tech. Quarterly, 1, I.

6 Chem. News, 6r, 5.

7 Chem. News. 58, I, $15,28$.

8 A nalyst, 10, 197.

- Bartram, Jour. Frank. Inst., March 17, 1891 , has published an article entitled " A Source of Error in the Determination of nitrates in Water by the Phenolsulphonic Acid Process," in which he says it was found that the phenolsulphonic acid undergoes a change after preparation. As ordinarily prepared, it may possibly undergo a change to the para acid; any difficulty is now obviated by the use of the pure disulphonic acid. I think the method has been submitted to an unusually severe test, 0.5 parts of nitrogen to 20 parts chlorine being very rarely met with in water analysis. It is unadvisable to estimate nitrates higher than 0.1 as it cannot be done with accuracy. Possibly the nitrate solution itself may have varied. I have solutions which contained I part of nitrogen as nitrates per $I \infty, \infty \infty$ and which in the course of seventy-two hours showed but 0.04 part, and in ninety-six hours none whatever. Another containing 4.5 parts, showed at the end of six days but 0.25 part.

$10 \mathrm{~J}$. A nal. Appl. Chem., $5,1$. 
their critique could be supported by experiment that this investigation was undertaken.

The process consists in evaporating the water,-usually ten cc.,-to dryness in a porcelain dish, treating the residue with phenolsulphonic acid, diluting with water and neutralizing with ammonia. In presence of nitrates, anmonium picrate is formed, the color of which is matched by standard solutions of potassium nitrate which have been treated in like manner.

One of the principal objections is the formation of the three nitrophenols "the principal products being the ortho and para mono nitrophenol." This cannot be the case: according to Richter, ${ }^{1}$ in the presence of a large excess of strong sulphuric acid-as we have here-the nitration goes farther, forming the di and tri-substituted compound. Granted, however, that the different amounts of these compounds were formed, it would be exceedingly anomalous to have under exactly the same conditions, at one time a large quantity of dinitrophenol formed, and at another time a large quantity of the trinitro body. Furthermore, the readings given in their comparative table of the various nitrophenols are probably incorrect; it has been found impossible to read colors as deep as 1.2 parts nitrogen per 100,000 in an eight inch tube more closely than twenty-five per cent.

The suggestion of one of them," of making up standards in eight inch tubes and allowing them to stand "for months," has been found to be of no advantage, as a very decided deterioration does take place. The other suggestion, of naking up standards by dilution of a solution obtained by treating a large quantity of potassium nitrate, is open to the objection that the colors produced are not the same as those from making up each one in a dish separately. In this connection I would remark that the same holds true of the colors produced by the Nessler reagent ; for example, ten $c c$. of standard ammonium chloride is made up to $100 \mathrm{cc}$. and the reagent added. The colors produced by diluting one cc., five cc., or ten cc. of this (colored) solution to fifty each are not the same as those obtained by diluting 0.2

1 Lehrbuch d. Org. Chemie., 5th Ed'n., 572.

2 Hazen, Report Mass. State Board of Health. "Purification of Sewage aud Water," $1890,7: 2$ 
cc., one cc., or two cc. of the standard ammonium chloride solution each to fifty cc. and adding the Nessler reagent, but in every case darker.

The process is dismissed with the remark: "We have not been successful in so controlling the reaction as to get a constant product." The chief agents in the action of nitric acid upon any compound are heat and concentration. If, therefore, the dishes containing the residues are always at the same temperature,which they must be, being at the temperature of the room-and the acid used of the same composition, it is difficult to see how the results could fail to agree in every case, and direct experi. ment proves this to be so, equal quantities of pure nitrate solutions agreeing exactly.

The investigation was conducted with reference to the following points :

I. AcIDs.
a. Various acids.
b. Method of treatment.
c. Quantity of acid used.

II. Evaporation.

d. Effect of sodium carbonate.

e. Temperature at which it is carried on.

f. Quantity evaporated.

g. Effect of chlorine.

III. CoMparison WITH STANDARDS.

The preparation of the phenolsulphonic acid is a matter of first importance, and for which, strange to say, no one has ever given definite directions. Kekulé found when phenol and sulphuric acid were mixed, according to the temperature, varying amounts of ortho and para phenol monosulphonic acids were formed. As these could not fail to influence the action upon the dry-water residue, an acid of perfectly definite composition,the pure disulphonic acid $\mathrm{C}_{6} \mathrm{H}_{8} \mathrm{OH}\left(\mathrm{SO}_{3} \mathrm{H}\right)_{2}$, probably $\mathrm{OH}$ : $\mathrm{SO}_{3}$ $\mathrm{H}: \mathrm{SO}_{3} \mathrm{H}=\mathrm{I}: 2: 4$, which with nitric acid gives picric acid even in the cold,$^{2}$-has been mostly used in these experiments.

This is prepared as follows: three grams of phenol of good quality and thirty-seven grams of pure sulphuric acid sp. gr.

1 Ztschr. für Chemie, 10, 199.

2 Kekule Lehrbuch III, 236. 
I.84 (Kahlbaum's synthetic phenol and Baker and Adamson's "Strictly Pure" sulphuric acid were usually employed) are mixed in a flask and heated for six hours in, not upon, a water bath to $100^{\circ}$

To determine the number of sulphonic groups, a portion of this mixture was neutralized with barium carbonate, lixiviated with water, filtered, the filtrate evaporated and the barium salt recrystallized, giving handsome orthorhombic prisms. A determination of barium in this gave 35.16 per cent., the theory requiring 35.22 per cent. A determination of water of crystallization gave 15.66 per cent., theory, 15.62 per cent. $\mathrm{H}_{2} \mathrm{O}$. The acid as thus prepared may crystallize out upons standing, it being rather insoluble in the strong sulphuric acid, in which it forms supersaturated solutions. It may be brought into solution by reheating a short tine, or by dilution with a small quantity of water, the undiluted acid has always been used.

The experiments were performed as follows: Two solutions were employed, one containing in one cc., O. I000 part of nitrogen per 100,000 as potassium nitrate; the other in addition to this seven parts chlorine as sodium chloride per 100,000. Of these, portions of one cc. each were measured out and diluted to five and ten cc. with distilled water and evaporated in porcelain dishes. $^{1}$

The $2 \frac{1}{2}$ inch evaporator containing the water residue was moistened with ten drops- $0.7 \mathrm{cc}$. - of the phenolsulphonic acid, and by rubbing with a short glass rod every part of the residue covered with it; seven cc. of water are now added,-neasured in later experiments by a Vanier" overflow pipette, - the solution stirred and three cc. anmonium hydrate, measured in like manner, added, the solution again stirred to insure uniformity, and the rods removed. In many cases the colors could be compared directly in the dishes, but in case of doubt the comparison took place in tubes about $1 \frac{5}{8}$ inches deep and $\frac{5}{8}$ inch in diameter.

1 A convenient way of marking these dishes is to remove a small patch of the glaze upon the ontside of the dish by glass etching ink, leaviug a rough surface for the lead pencil.

2J. A nal. Appl. Chem., 2, 145. 
Each determination is the average of at least two closely agreeing determinations.

All results are stated in parts per 100,000 .

I. ACIDS.

(a) VARIOUS ACIDS.

Equal parts. of phenol and The colors obtained were brown and an acsulphuric acids. curate matching was impossible.

Unknown mixture of ortho and, The colors obtained were fairly good, para sulphonic acids from five $\}$ but in some cases were reddish per cent. solution of phenol. $\int$ tinted.

Pure ortho phenol nono- $\}$ This gave a precipitate with the ammonia and sulphonic acid. $\left.{ }^{1}\right\}$ the colors were all bluish-green tinted.

Pure disulphonic acid, The colors obtained were a pure yellow and $7 \frac{1}{2}$ per cent. solution. $\}$ did not change upon standing. This, there$7 \frac{1}{2}$ per cent. solution. fore, gives the best results.

(b) METHOD OF TREATMENT.

At $100^{\circ}$.-Sprengel ${ }^{z}$ states that the residue should be nearly at $100^{\circ}$ when treated. Experiments performed by treating the residues upon the steam bath with the cold acid and also with the acid at $100^{\circ}$ gave no better results than those obtained in the ordinary way.

At $0^{\circ}$. - Treatment of the chilled residues upon ice with icecold acid gave decidedly lower results than those obtained in the usual manner, the nitration probably not going as far.

Addition of substances.-The addition of phenol or phenolsulphonic acid to the water before evaporation is of no advantage whatever.

\section{(c) Quantity of Acid Used.}

If chlorine be absent or in small quantity, the amount of acid used makes very little difference. Enough should be used to cover the residue readily, usually ten drops $=0.7 \mathrm{cc}$. is sufficient. For example, with equal quantities of the same nitrate solution which should have given a reading of 0.1000 , the following results were obtained:

1 Prepared by the cold evaporation in vacuo of the officinal solution of Merck's "Aseptol" and subsequent solution in sulphuric acid.

2 Pogg. Ann., 121, 188. 
TABLE I.
Showing the effect of the quantity of acid used. Without Chlorine. 10 drops acid. 6 drops.
$0.0925 \quad 0.0900$
With Chlorine, seven parts. ro drops acid. 6 drops.
$0.075^{\circ} \quad 0.0700$.

It is evident then that the use of too little acid causes the loss of nitric acid.

\section{EVAPORATION.}

It is well known that certain salts in solution undergo decomposition when boiled. Fittig' found this to be true of ammonium chloride, Debbits ${ }^{2}$ of several acetates with loss of acetic acid, and with the nitrate, sulphate, acetate and oxalate of ammonium with loss of the respective acids. Leeds ${ }^{3}$ and Fox ${ }^{4}$ have shown that nitrates are volatile at $100^{\circ}$ and even lower temperatures.

In order to áscertain what the influence of the evaporation really was, the following experiment was tried:

An intimate mixture of dry finely powdered potassium sulphate and nitrate was made by grinding them together in an agate mortar for several hours. Sixteen portions of roo milligrams each were accurately weighed out, each containing about 0.072 milligram of potassium nitrate. Twelve portions were dissolved each in ten cc. of water, four of these evaporated at $100^{\circ}$, four at $65^{\circ}$, and the remaining four at $20^{\circ}$ over sulphuric acid.

\section{TABLE II.}

Showing loss of nitrate by evaporation at various temperatures.

\begin{tabular}{|c|c|c|c|c|}
\hline & $\begin{array}{c}\text { Dry. } \\
\text { 0.I000 }\end{array}$ & $\begin{array}{c}20^{\circ} \\
0.0890\end{array}$ & $\begin{array}{c}65^{\circ} \\
0.0900\end{array}$ & $\begin{array}{c}100^{\circ} \\
\ldots \ldots\end{array}$ \\
\hline II $\ldots \ldots \ldots \ldots \ldots \ldots$ & 0.1050 & 0.1000 & 0.1080 & 0.1160 \\
\hline
\end{tabular}

The readings varied among themselves by 0.0200 and hence were averaged.

To obviate this variation, sixteen portions of one cc. each of a nitrate solution were measured out, nine cc. of water added and all allowed to evaporate over sulphuric acid at a temperature of $20^{\circ}$. Ten cc. of water were added to each one of twelve of

1 Ann. Chem. Pharm., 128, 189.

2 Ber. d. Chem. Ges., $5,820$.

8 Am.J. Sci., (3) 7, 197.

4 Tech. Quarteriy, 1, 1. 
these, and four evaporated at $20^{\circ}$, four at $65^{\circ}$, and four at $100^{\circ}$; this gave a series in which exactly the same amount of nitrate in every case was spread uniformly over the dishes and had been subjected to these various temperatures.

TABLE III.

Showing loss of nitrate by evaporation at various temperatures.

$\begin{array}{cccc}\text { Dry. } & 20^{\circ} . & 65^{\circ} . & 100^{\circ} . \\ 0.1000 & 0.0990 & 0.0965 & 0.0990\end{array}$

These results indicate that there is a loss of about one per cent. when the water is allowed to evaporate at ordinary temperatures over sulphuric acid, or in a steam bath where it evaporates rapidly and is immediately removed; at $65^{\circ}$ where the evaporation is slower, and the time of exposure to heat consequently lengthened, the loss is greater, in this case about three and a half per cent.

(d) EFFECT OF SODIUM CARBONATE.

With a view of fixing the nitric acid and thus diminishing the loss by evaporation, it has been suggested that carbonate of sodium be added to the water before evaporation; as carbon dioxide is given off upon treatment with acid, it is possible that this carries off with it a slight quantity of nitric acid. Ten portions each of nitrate solution, the reading of which should have been 0.1000 , were evaporated, the average reading was 0.0970 , with the addition of soda 0.0973 . Ten portions each of the same solution, containing in addition seven parts chlorine per 100,000 , when similarly treated gave 0.0821 , with the addition of soda 0.08 I 2 .

The avidity of acetic acid being much less than that of nitric acid, added to the fact that it was non-volatile at the temperatures employed, led to the suggestion that some of its salts be em. ployed as a substitute for the sodium carbonate. Sodium acetate when added to the water, gave rather lower results than those obtained when it was not used.

(e) TEMPERATURE AT WHICH EVAPORATION IS CARRIED ON.

The accompanying table shows clearly the effect of temperature upon the determination.

The solutions should have all read 0.1000 part nitrogen as 
potassium nitrate per 100,000 , they were all treated with car. bonate of sodium and each figure represents the mean of two closely agreeing determinations.

TABLE IV.

Effect of temperature.

Pure niter solution.

\begin{tabular}{cccc} 
& \multicolumn{3}{c}{ inantity evaporated. } \\
Temp. & $1 \mathrm{cc}$ & $5 \mathrm{cc}$. & rocc. \\
$100^{\circ}$. & 0.0925 & 0.0920 & 0.0920 \\
$65^{\circ}$. & 0.0890 & 0.0890 & 0.0880 \\
$20^{\circ}$. & 0.1050 & 0.0980 & 0.1000
\end{tabular}

Niter solution with 7.0 parts chlorine.

\begin{tabular}{cccc} 
& \multicolumn{3}{c}{ Quantity evaporated. } \\
lemp. & I cc. & $5 \mathrm{cc}$. & $10 \mathrm{cc}$. \\
$100^{\circ}$. & 0.0680 & 0.0700 & 0.0600 \\
$65^{\circ}$ & 0.0650 & 0.0650 & 0.0680 \\
$20^{\circ}$. & 0.0935 & 0.0920 & 0.0700
\end{tabular}

It is evident that there is a decided loss of nitrate when evaporation takes place at $65^{\circ}$ (the temperature which water assumes in a porcelain dish when upon the top of a water bath) over that lost at $100^{\circ}$ and here even over that lost at $20^{\circ}$. With chlorine this is even more perceptible; the reason for the increased loss at $65^{\circ}$ is probably, as already stated, that the time of exposure at this temperature is longer.

It is important, however, that the dishes be removed as soon as dry, as the subsequent exposure causes loss of nitrate.

TABLE V.

Effect of heat upon the dry residue.

Water contained 0.1000 part $\mathrm{N}$ as $\mathrm{N}_{2} \mathrm{O}_{5}, 7.0$ part $\mathrm{Cl}$. Quantity evaporated.

Removed at once........... $0.075^{\circ}$

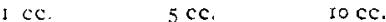

Exposed half an hour at $65^{\circ} \ldots 0.0700 \quad 0.0625 \quad 0.0690$

(f) QUANTITY OF SOLUTION EVAPORATED.

The quantity of solution evaporated seems to exercise but slight influence upon the results, although the smaller it is, the less the loss; this is evident from all three of the preceding tables.

(g) EFFECT OF CHLORINE.

The presence of large quantities of chlorides in the water does 
unquestionably lower the results. If the process be conducted in the usual way, by the evaporation of ten $c c$. at $100^{\circ}$ it shows in Table IV, a loss of from thirty to forty per cent. of nitrogen as nitrate. If a smaller quantity, five cc, or even one $c c$, be used, and the evaporation be carried on at $20^{\circ}$ over sulphuric acid, this loss is reduced to six or seven per cent., and of this probably one half is due to unavoidable errors in matching the colors.

If the chlorine amounts to but 2.0 parts per 100,000 , which is perhaps high in waters of doubtful character, it exercises practically no influence.

\section{TABIE VI.}

Effect of 2.0 parts of chlorine (as $\mathrm{NaCl}$ ) per $100, \infty 00$. Readings should be o. 1000 .

$\begin{array}{rll} & \text { I cc. } & \text { Io cc. } \\ 100^{\circ} . & 0.0900 & 0.0900 \text { (average of five determinations.) } \\ 65^{\circ} . & 0.0900 & 0.1000 \\ 20^{\circ} . & 0.0975 & 0.1050\end{array}$

\section{COMPARISON WITH STANDARDS.}

The yellow color of the picrate solution when as high as 0.1000 is extremely hard to match accurately; the tints must be read off at once without any delay as the difficulty increases with the time spent in comparison. It is well after reading perhaps a dozen, to rest the eyes by looking intently at a piece of blue paper.

As a result of a long series of experiments upon three different people,-all experts in the comparison,-it was found that the error of reading colors up to 0.0500 part $\mathrm{N}$ as $\mathrm{N}_{2} \mathrm{O}_{5}$ was 0.0020 or 0.0030 ; up to 0.1000 part $\mathrm{N}$ as $\mathrm{N}_{2} \mathrm{O}_{5}$ was 0.0050 .

No advantage was gained by the use of colored glasses or, when the colorimeters were used, of colored papers underneath them.

The process does not, as stated by Grandval and Lajoux, ${ }^{2}$ and also in Graham-Otto, ${ }^{2}$ estimate the nitrogen present as nitrites; this is clearly impossible, as the action of nitrous acid upon phenolsulphonic acid results in the formation of nitrosophenol

1 Compt, rend., ror, 62.

2 Lehrbuch d. anorgan. Chem., 2, Part I, 200. 
$\mathrm{C}_{6} \mathrm{H}_{4}, \mathrm{No}$ which is colorless in dilute solutions. This view was supported by an experiment with pure sodium nitrite prepared from pure silver nitrite, in which no color whatever was obtained upon treatment with the phenolsulphonic acid.

The results of this paper may be summarized as follows:

The most satisfactory manner of execution is, -in the case of a water high in nitrates, - to use that quantity which will give a reading of about 0.0700 when one or two cc. are employed; in the case of ground water ten cc. of a portion which has been clecolorized by the use of alumina in the cold, is evaporated upon the water bath, the dish being placed in the stean and removed as soon as dry, or better. when a drop is still remaining. In case the chlorine be high, which often accompanies high nitrates, this eraporation had best take place in racuo orer sulphuric acid, and the chlorine, if it exceeds 7.0 parts per 100,000. removed by silver sulphate which has been proved to be free from nitrate.'

In case one or two $\mathrm{cc}$. of the water is used the $0.7 \mathrm{cc}$. of acid used is sufficient to cover the residue completely,

The error of reading up to 0.0500 is 0.0020 or 0.0030 , four to six per cent. Ip to 0.1000 is 0.0040 or 0.0050 . four to five per cent., but the process as here described is more trustworthy, delicate and accurate than any process yet published.

For easy comparison 0.720 gram $\mathrm{KNO}_{3}$ can be dissolved in one liter of water, ten cc. of this allowed to evaporate in vacuo, treated with acid, made up to a liter and portions as wanted made alkaline with ammonia, of this ten $c c=0.1000$ part nitrogen as potassium nitrate per 100,000 .

Acknowlcdgnent. - The writer would hereby acknowledge his indebtedness to Prof. Drown and Mrs. E. H. Richards, chemists to the Massachusetts State Board of Health, for hints and suggestions, and to Miss Isabel F. Hyams and Mr. H. C. Tuttle, also of the Board of Health staff, for valuable assistance in making many of the readings involved in the preceding work.

DABORATORY OF SANITARY CHEMISTRY AND GAS ANALYSIS,

Mass. INSTITUTE OF ThehNOLOGY,

BOSTON, MASS

l Samples from f. Merck, marked "C. I'," contained enough nitrate to vitiate all resuits. 